\title{
TESTING MODELS FOR VARIATION IN INTELLIGENCE
}

\author{
L. J. EAVES \\ Department of Genetics, University of Birmingham, Birmingham, England B15 2TT

\section{SUMMARY} \\ A biometrical-genetical model for human variation adequately predicts \\ observed correlations for measured intelligence. The estimation procedure \\ is outlined.
}

Received 22.viii. 74

IN a recent review Jinks and Eaves (1974) stressed the close agreement between observed correlations between relatives for IQ and their expectations on the basis of a simple model. Their model involved five parameters: additive and dominant components of gene action $\left(D_{R}\right.$ and $\left.H_{R}\right)$; the marital correlation $(\mu)$; the correlation $(A)$ between spouses' additive genetical deviations; the component $\left(E_{c}\right)$ reflecting the environmental covariation of parent and offspring. The contribution of the environmental influences specific to individuals $\left(E_{1}\right)$ is a sixth parameter whose magnitude is fixed by the other five by the restraint that the total variance is unity.

Jencks' (1973) application of path coefficients to the analysis of intelligence was partly questioned because of what was believed to be an upper limit upon the value of the path between the genotypes of parent and offspring. No such limit exists in fact so his conclusions cannot be discounted on this basis. More critical, however, was Jinks and Eaves' re-analysis of published correlations (Burt, 1966; Jencks, 1973) which demonstrated that any signficant heterogeneity of heritability estimates obtained from different degrees of relationship can be removed if the contribution of dominance is precisely specified and a weighted least squares procedure is adopted.

The correlations analysed have already been tabulated (Jinks and Eaves, 1974), but details of the model and estimation procedure were necessarily omitted and are considered here. The model (table l) is that of Fisher (1918) for the correlations between relatives for a population in equilibrium under assortative mating with additions to specify the contribution of certain plausible environmental components. The model is a modification of that in the review because $\mu$ has been reparameterised in terms of $A$ and $D_{R}$. This restriction is necessarily imposed if all the assumptions in Fisher's model are to be tested adequately. It will be seen that although the restraint alters the estimates somewhat the fundamental interpretation remains unaltered.

In view of the fact that biometrical genetical models are usually linear and this model is substantially non-linear it is appropriate to outline the estimation procedure.

The model for our vector of observed correlations $\boldsymbol{x}$ may be written

$$
\boldsymbol{x}=f(\phi)+\boldsymbol{\varepsilon}
$$

where $\boldsymbol{f}(\boldsymbol{\phi})$ represents a vector of functions of the parameters $\phi$, and $\boldsymbol{\varepsilon}$ is a vector of deviations. We require $\phi$ to minimise the weighted sum of squared deviations

$$
\begin{aligned}
\chi^{2} & =\varepsilon^{\prime} \boldsymbol{W} \\
& =[x-f(\phi)]^{\prime} W[x-f(\phi)]
\end{aligned}
$$


$\boldsymbol{W}$ is the matrix of information about the observed statistics. Since the $\boldsymbol{x}$ are correlations, $W$ is a function of $f(\phi)$ which is unknown until $\phi$ has been estimated. Trial values for the elements of $f(\phi)$ have thus to be found so that an initial approximation to $W$ can be computed. It is convenient to use our observation vector $x$ as our trial estimate of $f(\phi)$. When $\phi$ has been estimated for a given estimate of $\boldsymbol{W}$ a closer approximation to $\boldsymbol{W}$ can be computed (Mather and Jinks, 1971). If the observations, $\boldsymbol{x}$, are independent, $\boldsymbol{W}$ is diagonal. This will be assumed here, but it may be the case, for Burt's data at least, that some individual observations have contributed to more than one correlation. The $i$ th diagonal element of $\boldsymbol{W}$ was set to $\left(n_{i}-2\right) /\left(1-f(\phi)_{\mathrm{i}}\right)^{2}$.

TABLE 1

Expectations for correlations between relatives

Correlation with

1. Parent living together (T)

2. Parent living apart (A)

3. Grandparent

4. Monozygotic twin T

5. Monozygotic twin A

6. Dizygotic twin $\mathrm{T}$ or full sibling $T$

7. Full sibling $A$

8. Uncle or aunt

9. 1st cousin

10. 2nd cousin

11. Foster parent $T$

12. Unrelated $T$

13. Spouse
Expectation

$\frac{1}{2}\left(1+\frac{2 A(1-A)}{D_{R}}\right)\left(\frac{1}{1-A}\right) D_{R}+E_{C}$

$\frac{1}{2}\left(1+\frac{2 A(1-A)}{D_{R}}\right)\left(\frac{1}{1-A}\right) D_{R}$

$\frac{1}{2}\left(1+\frac{2 A(1-A)}{D_{R}}\right)\left(\frac{1}{1-A}\right)\left(\frac{1+A}{2}\right)^{2} D_{R}$

$\frac{1}{2(1-A)} D_{R}+\frac{1}{4} H_{R}+E_{c}$

$\frac{1}{2(1-A)} D_{R}+1 H_{R}$

$\frac{1}{2}\left(\frac{1}{1-A}\right)\left(\frac{1+A}{2}\right) D_{R}+\frac{1}{16} H_{R}+E_{C}$

$\frac{1}{2}\left(\frac{1}{1-A}\right)\left(\frac{1+A}{2}\right) D_{R}+\frac{1}{16} H_{R}$

$\frac{1}{2}\left(\frac{1}{1-A}\right)\left(\frac{1+A}{2}\right)^{2} D_{R}+\frac{A}{32} H_{R}$

$\frac{1}{2}\left(\frac{1}{1-A}\right)\left(\frac{1+A}{2}\right)^{3} D_{R}+\frac{A^{2}}{64} H_{R}$

$\frac{1}{2}\left(\frac{1}{1-A}\right)\left(\frac{1+A}{2}\right)^{5} D_{R}+\frac{A^{4}}{256} H_{R}$

$E_{C}$

$E_{C}$

$\frac{2 A(1-A)}{D_{R}}$

Recognising that we can write $\boldsymbol{W}=\boldsymbol{V}^{\prime} \boldsymbol{V}$ we can recast (1) in the form:

$$
\chi^{2}=f^{\prime} f
$$

in which $\boldsymbol{f}=\boldsymbol{V}[\boldsymbol{x}-f(\boldsymbol{\phi})]$. If $\boldsymbol{W}$ is diagonal $\boldsymbol{V}=\boldsymbol{W}^{\mathrm{x}}$. We require $\boldsymbol{\phi}$ to set the gradient of $\chi^{2}$ to zero, i.e.

$$
\frac{\partial \chi^{2}}{\partial \phi}=2 J^{\prime} f=0
$$

where $J$ is the $m \times n$ matrix of the first partial derivatives of the $m$ weighted deviations, $f$, with respect to the $n$ parameters, $\phi$. 
Since the functions $f$ are non-linear in $\phi$ we must adopt an iterative procedure to obtain estimates for a given set of weights. The Newton-Raphson procedure ("Fisher's scoring method ") obtains a correction vector $\boldsymbol{\delta}_{0}$ by solving:

$$
\frac{1}{2} H_{0} \delta_{0}=-J_{0}^{\prime} f_{0} .
$$

Improved estimates $\phi_{1}$ are obtained as $\phi_{0}+\boldsymbol{\delta}_{0} . \quad \boldsymbol{H}_{0}$ is the $n \times n$ matrix of second partial derivatives of $\chi^{2}$ with respect to $\phi_{0}$. The procedure is repeated until $\boldsymbol{\delta}$ is sufficiently small. Exact evaluation of $\boldsymbol{H}$ can be tedious so an approximation of $\boldsymbol{H}$ may be used which forms the basis of the Gauss-Newton method. For $\boldsymbol{H}$ we substitute

$$
\boldsymbol{G}=2 J^{\prime} J
$$

which is equivalent to assuming that the functions of linear or that $f$ are small. $G$ may not be positive definite for trial values of $\phi$ and the process may not converge. Marquardt modified the method by introducing a parameter $\lambda>0$ and obtaining $\boldsymbol{\delta}$ as a solution of

$$
\left(\boldsymbol{J}^{\prime} \boldsymbol{J}+\lambda \boldsymbol{I}\right) \boldsymbol{\delta}=-\boldsymbol{J}^{\prime} \boldsymbol{f} .
$$

For sufficiently large $\lambda$ it has been shown that $\delta$ will always lead to $\phi$ which reduce $\boldsymbol{f}^{\prime} \boldsymbol{f}$. A further modification of this method has been implemented by the Nottingham Algorithms Group (1972) and the solutions reported here are obtained by this procedure.

Estimates of the parameters, together with an approximation, $\left(\boldsymbol{J}^{\prime} \boldsymbol{J}\right)^{-1}$, to their covariance matrix are given in table 2 for both sets of data. Inclusion

TABLE 2

Estimates of parameters and their covariance matrices

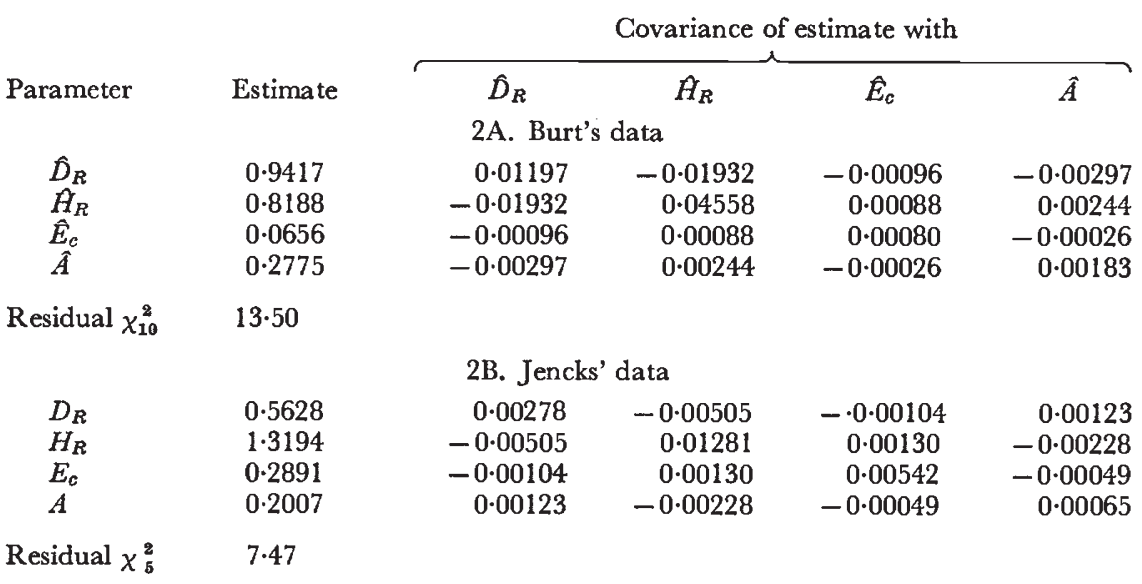

of the restraint leads to an increase in $\chi^{2}$ in both cases and to some changes in the parameters, but in neither case are the residuals significant and in neither case is there any need to alter the earlier conclusions about the magnitudes of the heritable and non-heritable components of variance, the mating system, or the kinds of gene action and their possible evolutionary basis. 
Jinks and Fulker (1970) assumed no environmental covariation of parent and offspring in their analysis of some of Burt's correlations, fitting instead of $E_{c}$ a component, $E_{2}$, which specified the environmental variation common to members of a sibship reared together. The expectations are the same as those in table 1 except that $E_{2}$ is substituted for $E_{c}$ and the environmental component does not appear in the expectations for statistics 1 and 2 . Fitting this model to the correlations (table 3) leaves little grounds for choice between models for Burt's data but reveals a much worse fit of the second model to Jencks' correlations.

TABLE 3

Parameters of Jinks' and Fulker's model

\begin{tabular}{|c|c|c|c|c|c|}
\hline \multirow[b]{2}{*}{ Parameter } & \multirow[b]{2}{*}{ Estimate } & \multicolumn{4}{|c|}{ Covariance of estimate with } \\
\hline & & $\hat{D}_{R}$ & $A_{R}$ & $\hat{E}_{2}$ & $\hat{A}$ \\
\hline $\begin{array}{l}D_{R} \\
H_{R} \\
E_{2} \\
A\end{array}$ & $\begin{array}{l}0 \cdot 9865 \\
0 \cdot 6229 \\
0 \cdot 0721 \\
0 \cdot 2889\end{array}$ & $\begin{array}{r}\text { 3A. Bur } \\
0.01321 \\
-0 \cdot 02058 \\
-0.00036 \\
-0.00396\end{array}$ & $\begin{array}{l}\text { ata } \\
-0.02058 \\
0.05197 \\
-0.00166 \\
0.00405\end{array}$ & $\begin{array}{r}-0.00036 \\
-0.00166 \\
0.00093 \\
-0.00015\end{array}$ & $\begin{array}{r}-0.00396 \\
0.00405 \\
-0.00015 \\
0.00193\end{array}$ \\
\hline Residual $\chi_{10}^{2}$ & \multicolumn{4}{|c|}{ 3B. Jencks' data } & \\
\hline $\begin{array}{l}D_{R} \\
H_{R} \\
E_{2} \\
A\end{array}$ & $\begin{array}{l}0 \cdot 8140 \\
0 \cdot 7882 \\
0 \cdot 1267 \\
0 \cdot 3660\end{array}$ & $\begin{array}{r}0.00072 \\
-0.00116 \\
-0.00026 \\
-0.00002\end{array}$ & $\begin{array}{r}-0.00116 \\
0.00901 \\
-0.00068 \\
-0.00019\end{array}$ & $\begin{array}{r}-0.00026 \\
-0.00068 \\
0 \cdot 00064 \\
-0.00028\end{array}$ & $\begin{array}{r}-0.00002 \\
-0.00019 \\
-0.00028 \\
0.00033\end{array}$ \\
\hline Residual $\chi_{5}^{2}$ & $120 \cdot 18$ & & & & \\
\hline
\end{tabular}

\section{Conclusions}

Successive improvements in the procedure by which biometricalgenetical models are fitted to correlations between relatives for IQ make little substantive difference to earlier conclusions about the statistical significance and biological importance of the various genetical and environmental determinants of individual differences in measured intelligence. The data may still be questioned, and other plausible models produced but the model considered here provides a criterion of simplicity and quality of fit which must be equalled by any alternative.

Acknowledgments.-The computations were conducted on the University of Birmingham ICL 1906A Computer. During the preparation of this paper, I enjoyed a prolonged and stimulating correspondence with A. S. Goldberger of the University of Wisconsin who has obtained estimates which differ from these only in detail. I am indebted to him for drawing my attention to a mistake in the expectation used for the avuncular correlations in the review paper.

\section{REFERENCES}

BURT, c. 1966. The genetic determination of intelligence, a study of monozygotic twins reared apart. British Fournal of Psychology, 57, 137-153.

FISHER, R. A. 1918. The correlation between relatives on the supposition of Mendelian inheritance. Transactions of the Royal Society of Edinburgh, 52, 399-433. 
JINKS, J. L., AND EAVES, L. J. 1974. IQ and inequality. Nature, 248, 287-289.

JINKS, J. L., AND FULKER, D. W. 1970. A comparison of the biometrical-genetical MAVA and the classical approaches to the analysis of human behaviour. Psychological Bulletin, 73, 311-349.

JENCKs, c. 1973. Inequality: A Reassessment of the Effect of Family and Schooling in America. Allen Lane, London.

MATHER, K., AND JINKS, J. L. 1971. Biometrical Genetics: The Study of Continuous Variation. Chapman and Hall, London.

NOTTINGHAM ALGORITHMS GROUP. 1972. EO4GAF. N.A.G. Library Manual, Document 427. 